\title{
New synchronization method for Plasmodium falciparum
}

\author{
Lisa C Ranford-Cartwright* , Abhinav Sinha, Georgina S Humphreys and Jonathan M Mwangi
}

\begin{abstract}
Background: Plasmodium falciparum is usually asynchronous during in vitro culture. Although various synchronization methods are available, they are not able to narrow the range of ages of parasites. A newly developed method is described that allows synchronization of parasites to produce cultures with an age range as low as 30 minutes.

Methods: Trophozoites and schizonts are enriched using Plasmion. The enriched late stage parasites are immobilized as a monolayer onto plastic Petri dishes using concanavalin A. Uninfected erythrocytes are placed onto the monolayer for a limited time period, during which time schizonts on the monolayer rupture and the released merozoites invade the fresh erythrocytes. The overlay is then taken off into a culture flask, resulting in a highly synchronized population of parasites.

Results: Plasmion treatment results in a 10- to 13-fold enrichment of late stage parasites. The monolayer method results in highly synchronized cultures of parasites where invasion has occurred within a very limited time window, which can be as low as 30 minutes. The method is simple, requiring no specialized equipment and relatively cheap reagents.
\end{abstract}

Conclusions: The new method for parasite synchronization results in highly synchronized populations of parasites, which will be useful for studies of the parasite asexual cell cycle.

\section{Background}

The human malaria parasite Plasmodium falciparum is usually asynchronous during in vitro growth [1,2], with all asexual stages of the parasite present. The generation of cultures containing highly synchronized parasites is necessary for studies of the cell cycle, for example, allowing accurate measurement of the lengths of different phases of the parasite life cycle.

Various synchronization methods have been published, which rely on removal of different parasite stages by differential osmotic lysis [3], physical separation relying on differential density [4-6] or by magnetic separation [7], temperature cycling [8], or cell cycle inhibitors [9]. Reviews of the available synchronization methods and their advantages and disadvantages have been published previously $[9,10]$. However all of these methods produce a population of parasites with a relatively wide age range -

\footnotetext{
*Correspondence: L.ranford-cartwright@bio.gla.ac.uk

${ }^{1}$ Division of Infection and Immunity, Faculty of Biomedical and Life Sciences, University of Glasgow, Glasgow Biomedical Research Centre, 120 University Place, Glasgow G12 8TA, Scotland

Full list of author information is available at the end of the article
}

the lowest reported is in the range of 3-5 hours $[6,10]$. An acknowledged problem is that narrowing of the range of age results in a reduction of parasitaemia.

A new method of synchronization has been developed by combining a recently published method to enrich cultures for later stage parasites using Plasmion (Laboratoire Fresenius Kabi, France) [11], with an inverted version of the "plaque assay" of J. Williams [12]. The enrichment method [11] is based on the slower sedimentation rate of late trophozoites and schizonts from $\mathrm{K}+$ (knob-expressing) strains [13] though a gelatin solution (Plasmagel), thus allowing their separation from earlier parasite stages and from uninfected erythrocytes [5]. Plasmion, a plasma substitute used in hospitals for hypovolaemia, is used in place of Plasmagel, which is no longer widely available [11]. This method allows the collection of merozoites within a user-specified window that can be as little as 30 minutes, or even less. The resultant culture contains infected erythrocytes with a very narrow age range, making this method very suitable for studies on cell cycle. 




Figure 1 Schematic of Plasmion enrichment.

\section{Methods}

Enrichment of late trophozoites and schizonts from in vitro cultures using Plasmion (Figure 1)

Asexual cultures of $P$. falciparum, grown according to standard protocols, were subjected to Plasmion treatment as previously described [11]. Typically, a culture was chosen with a relatively high proportion of later stage parasites with a parasitaemia of between 3 and 10\%. The culture material was centrifuged to pellet the red blood cells $(232 \times g$, 10 minutes $)$, the supernatant was removed, and the pellet was resuspended in fresh culture medium at a ratio of 3:1 medium: pellet. An equal volume of Plasmion (Laboratoire Fresenius Kabi, France) was added and the solution mixed and incubated at $37^{\circ} \mathrm{C}$ for 30 minutes. After incubation, the supernatant containing the older stages of the parasite (schizonts and trophozoites) was removed to a fresh tube, and the pellet containing the uninfected red blood cells and ring stage parasites was discarded. The collected supernatant was then centrifuged $(232 \times g$, 4 minutes $)$ to pellet the parasites, and the supernatant was discarded. A thin blood smear was made from the resultant pellet to measure the parasitaemia and to identify the parasite stages present. The cellular pellet was then resuspended in 1:10 pellet: incomplete medium (RPMI without serum), to give approximately $10 \%$ packed cell volume (PCV) for use on the monolayers.

\section{Formation of monolayers of parasitized erythrocytes using concanavalin A (Figure 2)}

$1.5 \mathrm{ml}$ of $10 \mu \mathrm{g} / \mathrm{ml}$ solution of concanavalin A (Sigma) was added to a sterile polystyrene tissue culture dish (diameter $35 \mathrm{~mm}$ ) and incubated for 30 minutes at $37^{\circ} \mathrm{C}$ [12]. Excess concanavalin A was removed and the dish washed with incomplete medium (RPMI without serum). $1.5 \mathrm{ml}$ of the Plasmion-enriched P. falciparum culture was added to the dish, placed into a modular incubator (Billups-Rothenberg, Inc), gassed (96\% $\mathrm{N}_{2}: 3 \% \mathrm{CO}_{2}: 1 \%$ $\mathrm{O}_{2}$ ) and incubated for 1 hour at $37^{\circ} \mathrm{C}$ to allow the erythrocytes to adhere to the concanavalin A.

Unbound erythrocytes were removed by aspiration and the monolayers were washed 3-5 times with incomplete RPMI medium to remove any unbound cells. The resul-



tant monolayer contained late trophozoite- and schizontinfected erythrocytes immobilized onto concanavalin A.

\section{Collection of synchronized ring stages from parasitized erythrocyte monolayers (Figure 2)}

$1.5 \mathrm{ml}$ of uninfected erythrocytes $(10 \% \mathrm{PCV}$ in complete RPMI) was laid over the monolayer, and the dish was then returned to the modular incubator, which was gassed and incubated at $37^{\circ} \mathrm{C}$ for the required time period (e.g. 30 minutes - 2 hours), depending on the range of ages required in the resultant culture. After this time the overlay cells were resuspended by gentle swirling, and collected and placed into a $25 \mathrm{~cm}^{2}$ tissue culture flask. Smears were taken from this flask immediately ("Time 1 hour" smear), and stained with Giemsa. $1 \mathrm{ml}$ of complete 
medium was added and the culture gassed as before, and incubated at $37^{\circ} \mathrm{C}$.

The monolayer was then washed 3-5 times with incomplete medium to remove any unbound cells, and was incubated with a further $1.5 \mathrm{ml}$ of uninfected erythrocytes at $10 \%$ PCV for the next hour. "Overlay 2" cells were collected after this period as before.

Repeated overlays could be applied to the same monolayer over the next 12 hours, and ring stage parasites collected over varying time periods to establish cultures of different ages and parasitaemia.

The "Time 1 hour" bloodsmear from each of the overlays was examined as soon as possible. Where there had been no or limited release of merozoites from mature schizonts in the incubation period, the resultant overlay culture had no or low ring stages present, and was discarded. The "Time 1 hour" smear was also discarded if there were parasites of any stage except for young ring stages. Such parasites probably indicate incomplete removal of unbound cells by the washing steps before incubation with uninfected erythrocytes.

\section{Results}

Enrichment of late trophozoites and schizonts from in vitro cultures using Plasmion

Plasmion treatment of asexual cultures produced an overall increase in parasitaemia of 4.2- to 7.7-fold (mean 5.8-fold) (Table 1). Both trophozoites and schizont stages were significantly enriched following Plasmion treatment, with a 6.0- to 14.3-fold increase in schizonts (mean 9.9-fold), and a 9.7- to 22-fold increase in trophozoites (mean 13.4-fold enrichment). A similar enrichment was seen for cultures of four different $P$. falciparum cloned lines (3D7, HB3, X12 and XP5).

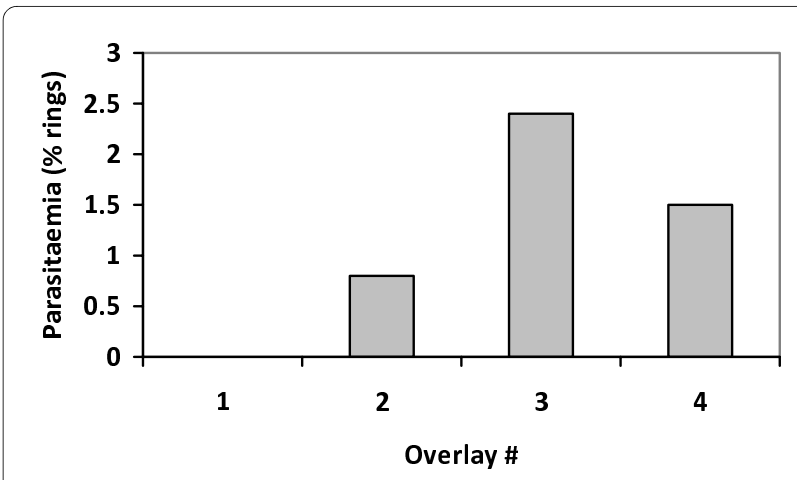

Figure 3 Example of overlay culture from one monolayer. A monolayer made from a Plasmion-enriched culture (XP5) at 66.8\% parasitaemia was overlaid with uninfected cells forming overlay\#1 for 1 hour. This was removed and replaced with fresh uninfected red cells for a further hour (overlay\#2), and then with a third replacement (overlay\#3) and finally a fourth (overlay \#4) for a final hour. There was no detectable parasitaemia in overlay \#1. Peak parasitaemia was observed in overlay \#3. Only ring stage parasites were seen in overlay \#2 to \#4.

\section{Collection of synchronized ring stages from parasitized erythrocyte monolayers}

Overlay cultures containing ring stage parasites only were obtained from the majority of monolayers, indicating successful synchronization. An example of a single monolayer and four overlay cultures is shown for parasite clone XP5 in Figure 3. The overall starting parasitaemia of the culture used to generate the monolayer, following Plasmion enrichment, was $66.8 \%$ (35\% schizonts: $29 \%$ trophozoites: line 5 in Table 1). This monolayer yielded a ring stage parasitaemia of $0.0 \%, 0.8 \%, 2.4 \%$ and $1.5 \%$ in successive one hour overlays beginning immediately after the establishment of the monolayer, indicating that there was no lysis of mature schizonts for first hour after the

Table 1: Plasmion enrichment of trophozoites and schizonts in P. falciparum cultures

\begin{tabular}{|c|c|c|c|c|c|c|c|c|c|c|c|c|}
\hline \multirow[t]{2}{*}{ Parasite } & \multicolumn{4}{|c|}{ Parasitaemia before Plasmion (\%) } & \multicolumn{4}{|c|}{ Parasitaemia after Plasmion (\%) } & \multicolumn{4}{|c|}{ Enrichment factor } \\
\hline & Total & $\mathbf{R}$ & $\mathbf{T}$ & $\mathbf{S}$ & Total & $\mathbf{R}$ & $\mathbf{T}$ & $\mathbf{S}$ & Total & $\mathbf{R}$ & $\mathbf{T}$ & $\mathbf{S}$ \\
\hline $3 \mathrm{D} 7$ & 11.3 & 6.1 & 2.4 & 2.8 & 47.2 & 2.8 & 26.1 & 18.3 & 4.2 & 0.5 & 11.1 & 6.5 \\
\hline $3 \mathrm{D} 7$ & 12.1 & 6.9 & 3.1 & 2.1 & 51.2 & 2.5 & 35.9 & 12.8 & 4.2 & 0.4 & 11.6 & 6.0 \\
\hline HB3 & 9.8 & 4.8 & 2.6 & 2.4 & 62.6 & 2.1 & 26.3 & 34.2 & 6.4 & 0.4 & 10.1 & 14.3 \\
\hline HB3 & 10.0 & 5.0 & 1.0 & 4.0 & 70.0 & 1.4 & 16.0 & 52.6 & 7.0 & 0.3 & 16.0 & 13.1 \\
\hline XP5 & 13.0 & 6.0 & 3.0 & 4.0 & 66.8 & 2.8 & 29.0 & 35.0 & 5.1 & 0.5 & 9.7 & 8.8 \\
\hline \multirow[t]{2}{*}{$\mathrm{X} 12$} & 10.2 & 4.0 & 1.1 & 5.1 & 78.1 & 2.1 & 22.0 & 53.9 & 7.7 & 0.5 & 22.0 & 10.8 \\
\hline & & & & & & & \multicolumn{2}{|c|}{ Mean enrichment } & 5.78 & 0.4 & 13.4 & 9.9 \\
\hline
\end{tabular}

Asexual culture parasitaemia was estimated by examination of 2,000 erythrocytes for each culture, either immediately before or following enrichment with Plasmion. The numbers of ring (R), trophozoite (T) and schizont (S) stages present in each smear were also recorded. Trophozoites were distinguished from ring stage parasites by the appearance of pigment. Schizonts were distinguished from trophozoites by the presence of two or more nuclei. The enrichment factor was calculated by dividing the post-Plasmion treatment parasitaemia by that at pretreatment. 
monolayer establishment, followed by a peak of schizont lysis 3-4 hours post monolayer establishment. The rings present in these one-hour cultures represent merozoites emerging from schizonts and reinvading fresh red cells within a one hour window, and are thus highly synchronized.

\section{Discussion}

A simple new method is described to obtain highly synchronized asexual cultures of $P$. falciparum suitable for studies of the asexual cell cycle. Plasmion treatment [11] of the asynchronous culture increases the overall parasitaemia approximately six-fold, by the removal of uninfected and ring-stage infected red blood cells, and substantially enriches the culture for schizonts (approximately ten-fold) and trophozoites (approximately 13fold). An overall decrease of ring stage parasites was also observed (Table 1). The efficiency of the method does not require starting cultures with high numbers of more mature parasites.

The enriched culture is used to create a monolayer of infected cells bound to a plastic Petri dish using the lectin concanavalin A. In the original description of the "Plaque assay" [12], uninfected erythrocytes are used to form the monolayer, and the overlay consists of parasitized red cells. Merozoites released from the overlay invaded the monolayer. Here the method has been inverted, forming the monolayer from parasitized erythrocytes, and the overlay of uninfected red cells. Mature schizonts in the monolayer release their merozoites which invade uninfected red cells in the overlay. The overlay is then removed to establish new asexual cultures. By limiting the time of contact between the monolayer and overlay, merozoites invade within a narrow time-window, which can be as short as 30 minutes, and could theoretically be shorter than this. The overlay culture thus represents a highly synchronized population of parasites.

The degree of synchronicity of the overlay culture can be changed by varying the length of time the overlay and monolayer remain in contact. Longer periods of time generally result in a higher parasitaemia, but with a less tightly synchronized parasite population. The initial Plasmion treatment increases the monolayer parasitaemia to greatly in excess of that normally possible in vitro, producing a higher parasitaemia in the overlay culture than would be possible without the use of enrichment. Thus it is possible to narrow the age range while maintaining a useful parasitaemia.

The chief difficulty with the method is to judge when the overlay should be placed onto the monolayer, at the period of maximum schizont rupture. The appropriate time can be estimated from the number and size of schizonts in the smear taken of the Plasmion-treated material. A single monolayer can be used to collect multiple overlay cultures (the maximum prepared is five one-hour overlays, but theoretically many more could be taken). In addition, in this paper a $2.6 \mathrm{~cm}$ diameter dish was used, but larger dishes could be substituted if larger volumes of synchronized culture were required.

In comparison to other available methods of synchronization, the new method is simple and cheap, requiring no specialized equipment such as magnetic columns [7] or complicated density gradients [14].

\section{Conclusions}

By combining two existing techniques, a new method is presented that allows very tight synchronization of cultures compared to other published methods, down to a time window of 30 minutes (or less) if required.

\section{Competing interests}

The authors declare that they have no competing interests.

\section{Authors' contributions}

LCRC conceived the technique, designed the study, participated in the data analysis and drafted the paper. GSH optimized the Plasmion conditions and reviewed the manuscript critically. JMM and AS optimized and carried out the monolayer-based synchronizations, participated in the analysis of the results and reviewed the manuscript critically. JMM helped to draft the final manuscript. All authors read and approved the final manuscript.

\section{Acknowledgements}

We thank Mrs. Elizabeth Peat for expert technical assistance, and Dr. Françoise Benoit-Vical, Service de Parasitologie et Mycologie, Hôpital Rangueil-Larrey CHU Toulouse, France, for the kind gift of Plasmion. GSH is supported by a BBSRC PhD Studentship. AS was supported by a Chevening Scholarship. JMM is supported by the Wellcome Trust [grant number 078749].

\section{Author Details}

Division of Infection and Immunity, Faculty of Biomedical and Life Sciences, University of Glasgow, Glasgow Biomedical Research Centre, 120 University Place, Glasgow G12 8TA, Scotland

Received: 9 October 2009 Accepted: 17 June 2010

Published: 17 June 2010

\section{References}

1. Trager W, Jensen JB: Human malaria parasites in continuous culture. Science 1976, 193:673-675.

2. Haynes JD, Diggs CL, Hines FA, Desjardins RE: Culture of human malaria parasites Plasmodium falciparum. Nature 1976, 263:767-769.

3. Lambros C, Vanderberg JP: Synchronization of Plasmodium falciparum erythrocytic stages in culture. J Parasitol 1979, 65:418-420.

4. Aley SB, Sherwood JA, Howard RJ: Knob-positive and knob-negative Plasmodium falciparum differ in expression of a strain-specific malarial antigen on the surface of infected erythrocytes. J Exp Med 1984, 160:1585-1590.

5. Jensen JB: Concentration from continuous culture of erythrocytes infected with trophozoites and schizonts of Plasmodium falciparum. Am J Trop Med Hyg 1978, 27:1274-1276.

6. Mrema JE, Langreth SG, Jost RC, Rieckmann KH, Heidrich HG: Plasmodium falciparum: isolation and purification of spontaneously released merozoites by nylon membrane sieves. Exp Parasitol 1982, 54:285-295.

7. Ahn SY, Shin MY, Kim YA, Yoo JA, Kwak DH, Jung YJ, Jun G, Ryu SH, Yeom JS, Ahn JY, Chai JY, Park JW: Magnetic separation: a highly effective method for synchronization of cultured erythrocytic Plasmodium falciparum. Parasitol Res 2008, 102:1195-1200.

8. Haynes JD, Moch JK: Automated synchronization of Plasmodium falciparum parasites by culture in a temperature-cycling incubator. Methods Mol Med 2002, 72:489-497. 
9. Naughton JA, Bell A: Studies on cell-cycle synchronization in the asexual erythrocytic stages of Plasmodium falciparum. Parasitology 2007, 134:331-337.

10. Hoppe HC, Verschoor JA, Louw Al: Plasmodium falciparum: a comparison of synchronisation methods for in vitro cultures. Exp Parasitol 1991, 72:464-467.

11. Lelievre J, Berry A, Benoit-Vical F: An alternative method for Plasmodium culture synchronization. Exp Parasitol 2005, 109:195-197.

12. Inselburg J: Gametocyte formation by the progeny of single Plasmodium falciparum schizonts. J Parasitol 1983, 69:584-591.

13. Pasvol G, Wilson RJ, Smalley ME, Brown J: Separation of viable schizontinfected red cells of Plasmodium falciparum from human blood. Ann Trop Med Parasitol 1978, 72:87-88.

14. Nillni EA, Londner MV, Spira DT: A simple method for separation of uninfected erythrocytes from those infected with Plasmodium berghei and for isolation of artificially released parasites. Z Parasitenkd 1981 64:279-284

doi: $10.1186 / 1475-2875-9-170$

Cite this article as: Ranford-Cartwright et al., New synchronization method for Plasmodium falciparum Malaria Journal 2010, 9:170

Submit your next manuscript to BioMed Central and take full advantage of:

- Convenient online submission

- Thorough peer review

- No space constraints or color figure charges

- Immediate publication on acceptance

- Inclusion in PubMed, CAS, Scopus and Google Scholar

- Research which is freely available for redistribution

Submit your manuscript at www.biomedcentral.com/submit
C) Biomed Central 